\title{
Work-distribution quantumness and irreversibility when crossing a quantum phase transition in finite time
}

\author{
Krissia Zawadzki $\odot,{ }^{1,2}$ Roberto M. Serra, ${ }^{3}$ and Irene D'Amico $\odot^{2,4,5}$ \\ ${ }^{1}$ Department of Physics, Northeastern University, Boston, Massachusetts 02115, USA \\ ${ }^{2}$ Departamento de Física e Ciência Interdisciplinar, Instituto de Física de São Carlos, University of São Paulo, \\ Caixa Postal 369, 13560-970 São Carlos, São Paulo, Brazil \\ ${ }^{3}$ Centro de Ciências Naturais e Humanas, Universidade Federal do ABC, Avenida dos Estados 5001, \\ 09210-580 Santo André, São Paulo, Brazil \\ ${ }^{4}$ Department of Physics, University of York, York YO10 5DD, United Kingdom \\ ${ }^{5}$ International Institute of Physics, Federal University of Rio Grande do Norte, Natal, Brazil
}

(Received 18 August 2019; revised 20 December 2019; accepted 7 July 2020; published 29 July 2020)

\begin{abstract}
The thermodynamic behavior of out-of-equilibrium quantum systems in finite-time dynamics encompasses the description of energy fluctuations, which dictates a series of the system's physical properties. In addition, strong interactions in many-body systems strikingly affect the energy-fluctuation statistics along a nonequilibrium dynamics. By driving transient currents to oppose the precursor to the metal-Mott-insulator transition in a diversity of dynamical regimes, we show how increasing many-body interactions dramatically affect the statistics of energy fluctuations and, consequently, the extractable work distribution of finite Hubbard chains. Statistical properties of such distributions as its skewness with its impressive change across the transition can be related to irreversibility and entropy production. Even for slow driving rates, the quasi quantum phase transition hinders equilibration, increasing the process irreversibility, and inducing strong features in the work distribution. In the Mott-insulating phase, the work fluctuation-dissipation balance gets modified with the irreversible entropy production dominating over work fluctuations. Because of this, effects of an interaction-driven quantum phase transition on thermodynamic quantities and irreversibility must be considered in the design of protocols in small-scale devices for application in quantum technology. Eventually, such many-body effects can also be employed in work extraction and refrigeration protocols on a quantum scale.
\end{abstract}

DOI: 10.1103/PhysRevResearch.2.033167

\section{INTRODUCTION}

After more than a century, the increasing availability of nanoscale technologies has challenged the community to develop the well-established laws of thermodynamics beyond the so-called thermodynamic limit [1-7]; quantum thermodynamics is now extending concepts, such as heat, work, and entropy to small few-particle quantum systems $[1,8,9]$. At the same time, working conditions for quantum technology devices often correspond to finite temperatures and nonequilibrium regimes [10] so that the development of related formalism is in high demand. In quantum systems, thermodynamic probability distributions contain rich information about the possible transitions between eigenstates [11] and, more interestingly, thermal and quantum fluctuations [12-14] and equilibration and irreversibility [15-18]. Identification of nonclassical features in work and heat distributions of the

Published by the American Physical Society under the terms of the Creative Commons Attribution 4.0 International license. Further distribution of this work must maintain attribution to the author(s) and the published article's title, journal citation, and DOI. quantum system is a topic under investigation with, thus far, some interesting results for harmonics oscillators $[19,20]$.

Quantum phase transitions (QPTs) are an exquisitely quantum phenomenon, so there is interest in studying their signatures on quantum thermodynamic quantities and their distributions (fluctuations) [14,16,18,21-28]. In addition, many-body interactions, which are ubiquitous and notoriously difficult to treat, assume an even more complex role in out-of-equilibrium quantum systems $[29,30]$ where, e.g., they may affect the way the system reaches or settles into different phases. Relevant questions are as follows: what is the role of many-body interactions for quantum particles driven out of equilibrium, and how do they affect quantum thermodynamical quantities? Do they contribute or oppose reversibility [31] and thermalization? What if many-body interactions induce a QPT, what signatures appear in thermodynamic distributions? And how do they depend on the system size?

Most of the previous studies of QPT signatures in quantum thermodynamics focused on QPTs driven by external fields and/or on the sudden quench regime. They analyzed features of quantum thermodynamic quantities, sometimes up to the second moment of their distribution, and their evolution as the critical parameter, usually an external field, is (suddenly) driven across the transition. 
In this paper, we consider the above questions in the context of microscopic models for strongly correlated systems undergoing finite-time processes at finite temperatures. With state-of-the-art simulations, we study the nonhomogeneous one-dimensional Hubbard model at half-filling as it is driven out of equilibrium. Finite Hubbard chains may undergo a precursor to the metal-Mott-insulator transition, a QPT driven solely by many-body interactions. Considering the out-ofequilibrium work probability distribution and its statistics, we inspect the first three moments, related to the mean, variance, and skewness. The latter has been to a large extent overlooked, and we demonstrate that it allows to characterize the transition between the different coupling regimes, including the precursor to the metal-Mott-insulator QPT (pM-QPT) as well as the different dynamical regimes (sudden quench to nearly adiabatic). Our results also demonstrate that, by considering the sudden quench regime alone, one misses the contribution of the dynamics to the QPT signatures, which becomes dominant in finite-time regimes. Many-body interactions strikingly affect the shape of the work probability distribution: Whereas it acquires a bell shape for increasing system size and weak interactions, this feature is completely dismantled by the pMQPT, which also averts the system from equilibrium. Interestingly, we show that, in the Mott-insulating phase, entropy production dominates over work fluctuations, in contrast to the literature [32-34]. Finally, we relate the skewness with the entropy production and propose its role as a witness of irreversibility for many-body systems out of equilibrium.

\section{DRIVEN INHOMOGENOUS HUBBARD CHAINS}

The Hubbard model allows for both itinerant electron spins (conduction band) and localized magnetic moments. It was initially designed to describe strongly correlated systems, such as transition metals; more recently it has been utilized to describe systems of importance to quantum technologies, such as cold atoms in an optical lattice, chains of trapped ions, excitons, electrons in coupled quantum dots, or small molecules [35-40]. Even nondriven, short Hubbard chains are characterized by a very rich physical behavior with manybody interactions driving a precursor to the metal-to-Mottinsulator transition [41-43], and studies of a driven Hubbard dimer show promising results $[44,45]$.

Here, we consider half-filled fermionic chains undergoing a process in which a time-dependent electric field is applied for a finite time, ranging from fast to close-to-adiabatic dynamics. Their Hamiltonian is $H(t)=$ $-J \sum_{j=1}^{L-1}\left(\hat{c}_{j, \sigma}^{\dagger} \hat{c}_{j+1, \sigma}+\hat{c}_{j+1, \sigma}^{\dagger} \hat{c}_{j, \sigma}\right)+U \sum_{j=1}^{L} \hat{n}_{j \uparrow} \hat{n}_{j \downarrow}+$ $\sum_{j=1}^{L} V_{j}(t) \hat{n}_{j \sigma}$, where $\hat{c}_{j \sigma}^{\dagger}\left(\hat{c}_{j \sigma}\right)$ are the creation (annihilation) operators for a fermion with spin $\sigma=\uparrow, \downarrow$ in the $j$ th site, $\hat{n}_{j \sigma}=\hat{c}_{j \sigma}^{\dagger} \hat{c}_{j \sigma}$ represents the corresponding $j$-site occupation, $J$ is the hopping parameter, $U$ is the Coulomb on-site repulsion, and $V_{j}(t)=\Delta_{j} t / \tau$ with $\Delta_{j}=\frac{10 J}{L-1} j$ is the time-dependent linear potential that drives an out-of-equilibrium transient current along the chain.

The system is initially in thermal equilibrium at temperature $\beta^{-1}=k_{B} T=2.5 \mathrm{~J}$ where not otherwise stated (where $k_{B}$ is the Boltzmann constant and $T$ is the absolute temperature) with $\rho(t=0)=e^{-\beta H(t=0)} / Z_{t=0}$, and $Z_{t=0}=\operatorname{Tr}\left[e^{-\beta H(t=0)}\right]$.
The driving time $\tau$ controls the rate of the dynamics that steers $H_{0}=H(t=0)$ to $H_{f}=H(t=\tau)$. The final Hamiltonian $H_{f}$ is independent of $\tau$. Our results were obtained via exact diagonalization; the time evolution was calculated by a routine provided by the QUTIP package [46].

\section{STATISTICS OF WORK AND MANY-BODY INTERACTIONS}

The probability distribution characterizing the work [8] performed on the closed quantum system ${ }^{1}$ is given by

$$
P(W)=\sum_{n, m} p_{n}^{0} p_{m \mid n}^{\tau} \delta\left[W-\left(\epsilon_{m}^{\tau}-\epsilon_{n}^{0}\right)\right],
$$

where $p_{n}^{0}$ is the initial-state occupation probability of the $n$th eigenstate $|n\rangle$ of energy $\epsilon_{n}^{0}$ of $H_{0}$, and $p_{m \mid n}^{\tau}$ is the conditional probability for $|n\rangle$ to make a transition to the $m$ th eigenstate $|m\rangle$ of $H_{f}$. After the unitary driving, the system will eventually interact with the environment and get thermalized again.

The complexity of $P(W)$ scales with the number of the possible energy transitions. In the systems we consider, halffilling with zero magnetization, the number of allowed transitions increases from 16 for $L=2$ to $2.4 \times 10^{7}$ for $L=8$. $^{2}$ This is highlighted by Figs. 1 (a) and $1(\mathrm{c})$, where $P(W)$ is shown for $L=4$ and $L=8$ for the noninteracting case ( $U=$ $0)$. The exponential increase in the number of transitions transforms the distribution from an irregular set of peaks to a bell shape: ${ }^{3}$ changes in the type of dynamics-from (quasi) sudden quench ( $\tau=0.1$ ) to close-to-adiabatic behavior ( $\tau=$ $10 / J)$ - strongly affect the shape of the distribution, which becomes increasingly asymmetric as $\tau$ increases. On the contrary, when considering the strongly interacting regime $(U=$ $10 J)$, Figs. 1(b) and $1(\mathrm{~d})$, the shape of $P(W)$ seems basically unaffected (see the Supplemental Material for animations [47]). We attribute this behavior to the insulating phase which de facto substantially reduces the available Hilbert space by drastically reducing the probability of most potential transitions during the dynamics.

This qualitative picture is quantified by the $k$ th central moments of the work distribution $P(W)$,

$$
\overline{\mathcal{W}}^{k}=\left\langle(W-\bar{W})^{k}\right\rangle=\sum_{i} P\left(W_{i}\right)\left(W_{i}-\bar{W}\right)^{k} .
$$

The moments $k=1$ (mean), $k=2$ (variance), and $k=$ 3 (skewness) are shown in Fig. 2, $L=4$ left and $L=8$ right; the corresponding "heatmaps" for $k=3$ is in Fig. 3 where the white line indicates $\overline{\mathcal{W}}^{3}=0$ (see the Supplemental Material for other heatmaps [47]). The first three moments are strongly dependent on $\tau$ for weak interactions $U<J$,

\footnotetext{
${ }^{1}$ We consider processes fast enough to be represented as (a unitary) closed system dynamic. In other words, the calculations correspond to the scenario where the time duration of the driving protocol $\tau$ is much smaller than any decoherence or relaxation times.

${ }^{2}$ There are 4900 spin configurations for $L=8$ having $S_{z}=0$. Because of the finite-time dynamics and finite temperatures, it is currently not possible to treat numerically exactly larger systems.

${ }^{3}$ We obtain similar results for $U \lesssim J$. See the distributions $P(W)$ for $L=4,6,8$ and $U=J$ in the Supplemental Material [47].
} 

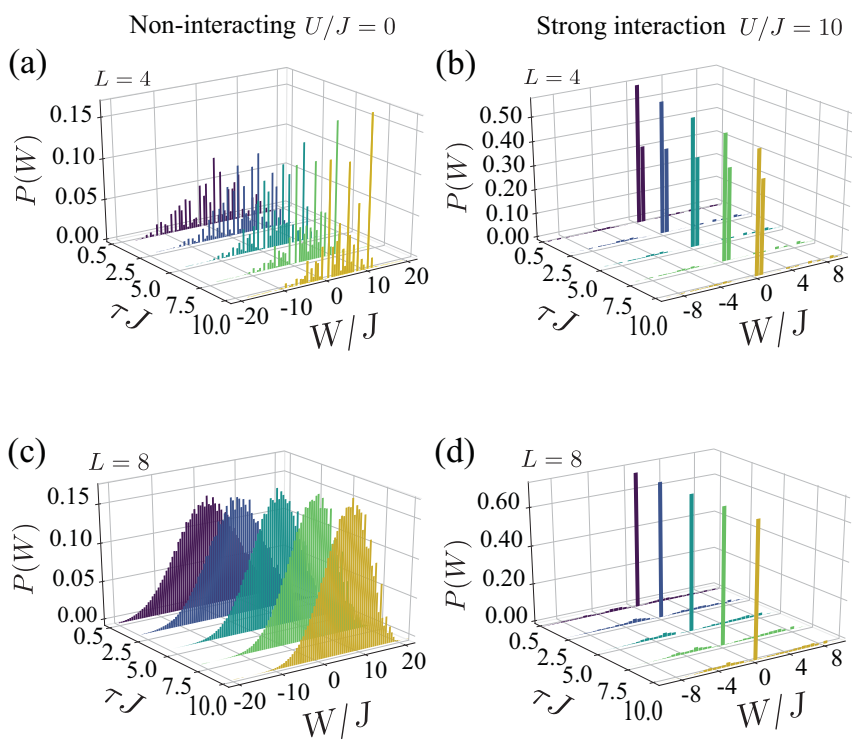

FIG. 1. Work distribution $P(W)$ for fermionic Hubbard chains at half-filling driven by a time-dependent electric potential difference. Panels (a) and (b) refer to four-site chains, whereas (c) and (d) refer to eight sites. The left panels (a) and (c) show the noninteracting case $(U=0)$, and the right panels (b) and (d) show the strong-interaction regime $(U=10 J)$. Each panel displays $P(W)$ for different driving times, from quasi-sudden-quench $(\tau=0.5 / J)$ to a close-to-adiabatic $(\tau=10 / J)$ dynamics.

whereas almost $\tau$ independent for $U \approx 10 J$, once interactions have driven the pM-QPT and the system becomes quasiinsulating. ${ }^{4}$ Regardless of the huge increase in the Hilbert space, the behavior across the transition is qualitatively independent from the system size, hinting to a possible scaling behavior. The most striking features appear in the skewness $\overline{\mathcal{W}}^{3}$. For sudden quenches $\tau \ll J^{-1}$, the skewness is relatively small and depends only weakly on $U$ (see Fig. 3). However, for finite-time processes $\tau \gtrsim 0.5 / J, \bar{W}^{3}$ changes sign across the pM-QPT (white line in Fig. 3) with proper minima and maxima bracketing the transition when $\tau \gtrsim 2.5 / J$ (see Figs. 3 and lower panels of Fig. 2). As $U$ increases, the system suffers a dynamic competition between the transient current induced by the drive and the increasing on-site repulsion. This leads to a dramatic change in the shape of $P(W)$ with a marked asymmetry shifting from left (before the pM-QPT) to right (after the pM-QPT). As $\tau$ increases, the region inbetween $\overline{\mathcal{W}}^{3}(U)$ extrema shifts towards larger $U$ 's (see Fig. 2, lower panels). We observe that the strong asymmetry in the distribution and a dramatic change in this asymmetry signals an exquisitely quantum phenomenon, such as a QPT.

\footnotetext{
${ }^{4}$ In this phase, the energy gap between any states composed solely by spin configurations with singly occupied sites, among which the ground state is included, is much lower than the energetic difference between any of these states and the first excited state composed by components having double occupied sites.
}

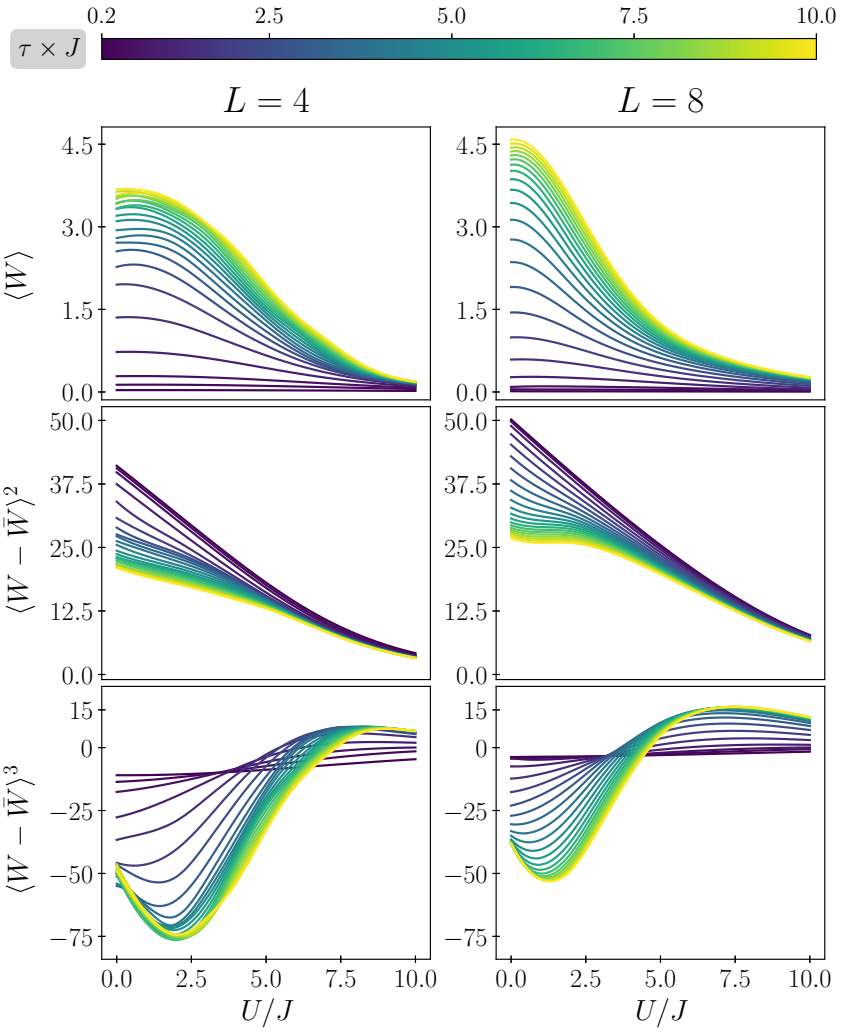

FIG. 2. First three moments of the work distribution (as labeled) versus $U$ for $0.2 / J \leqslant \tau \leqslant 10 / J$ and chain length $L=4$ (left) and $L=8$ (right).

\section{ENTROPY PRODUCTION AND IRREVERSIBILITY}

Together with the statistics of work, we can inspect how the pM-QPT affects irreversibility. We quantify this by considering the entropy production $[15,48-50]$,

$$
\langle\Sigma\rangle=S\left(\rho_{\tau} \| \rho_{\tau}^{\mathrm{eq}}\right),
$$

where $S\left(\rho_{\tau} \| \rho_{\tau}^{\mathrm{eq}}\right)=\operatorname{Tr} \rho_{\tau}\left(\ln \rho_{\tau}-\ln \rho_{\tau}^{\mathrm{eq}}\right)$ defines the Kullback relative entropy between the final-state $\rho_{\tau}=\mathcal{U}_{\tau} \rho_{0}^{\text {eq }} \mathcal{U}_{\tau}^{\dagger}$ and its equilibrium counterpart $\rho_{\tau}^{\mathrm{eq}}=e^{-\beta H(t=\tau)} / Z_{t=\tau}$ with $\mathcal{U}_{t}$ as the time-evolution operator. We note that $\langle\Sigma\rangle / \beta$ corresponds also to the energy that would be dissipated if thermalization would follow the finite-time driven protocol. We
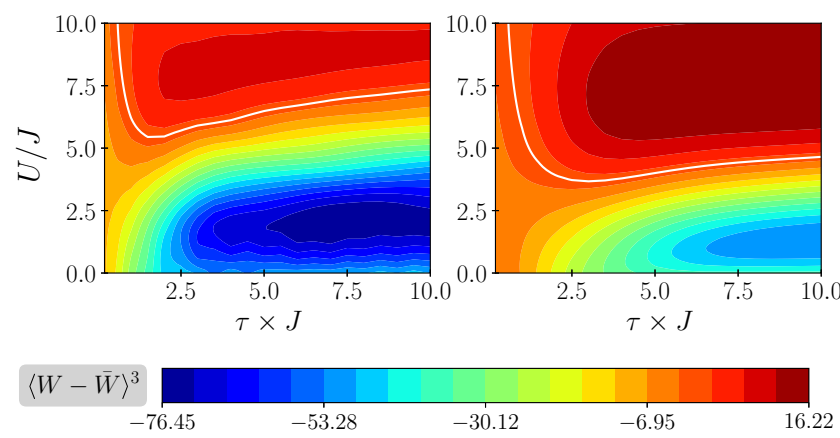

FIG. 3. Heatmaps of the skewness of the work distribution, for $L=4$ (left) and $L=8$ (right). The white line indicates $\overline{\mathcal{W}}^{3}=0$. 

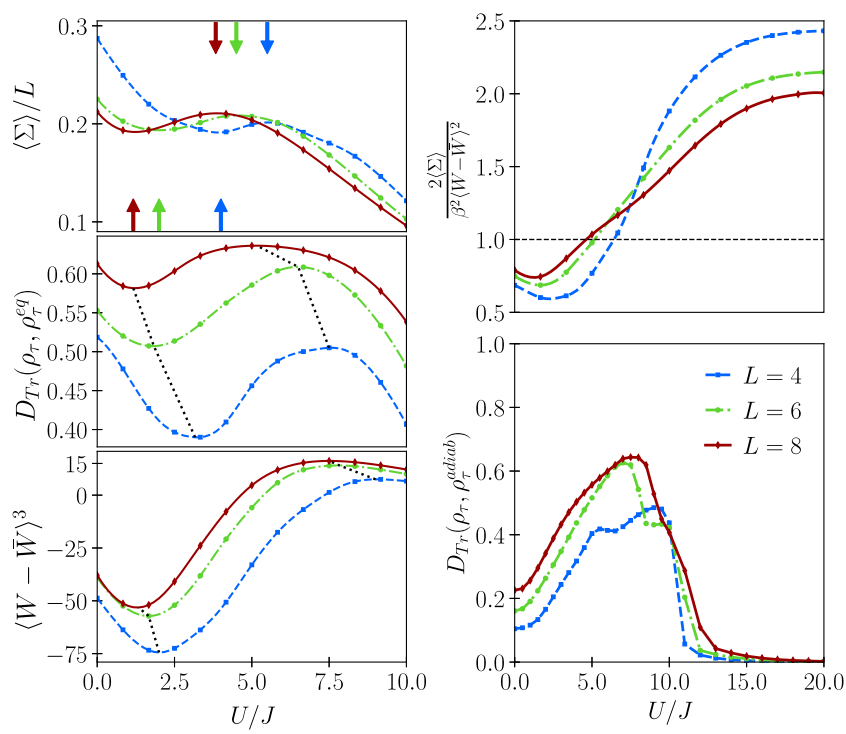

FIG. 4. Left panels: Scaled entropy production $\langle\Sigma\rangle / L$ (top), trace distance $D_{\mathrm{Tr}}\left(\rho_{\tau}, \rho_{\tau}^{\mathrm{eq}}\right)$ (middle), and skewness $\langle W-\bar{W}\rangle^{3}$ (bottom), versus coupling strength $U / J$ and for chains of size $L=4,6,8$ and $\tau \times J=10$. The arrows (top panel) and the dashed black lines (middle and bottom) connect minima and maxima for increasing system size. Right panels: entropy production to work fluctuations ratio versus coupling strength $U / J$ for chains of size $L=4,6,8$ and $\tau \times J=10$ (top). Trace distance $D_{\operatorname{Tr}}\left(\rho_{\tau}, \rho_{\tau}^{\text {adiab }}\right)$ between the final and the corresponding adiabatic states; same parameters as for the upper panel (bottom).

examine the entropy production in our systems in various dynamical and coupling regimes, full results for $L=4$ and $L=8$ are reported in the Supplemental Material [47].

For a finite quantum system, adiabaticity in the quantum dynamics does not imply, in general, equilibration, hence, to quantitatively investigate this discrepancy, we focus on large $\tau$ results and use in addition to $\langle\Sigma\rangle$ the trace distance [51] between the final and the corresponding equilibrium states $D_{\operatorname{Tr}}\left(\rho_{\tau}, \rho_{\tau}^{\mathrm{eq}}\right)=\operatorname{Tr}\left[\sqrt{\left(\rho_{\tau}-\rho_{\tau}^{\mathrm{eq}}\right)^{\dagger}\left(\rho_{\tau}-\rho_{\tau}^{\mathrm{eq}}\right)}\right] / 2 .^{5}$ This is plotted in the middle left panel of Fig. 4, together with $\langle\Sigma\rangle / L$ (top left) and the skewness (bottom left) as a function of $U / J$ for $\tau \times J=10$ and $L=4,6,8$. For all the system sizes studied, all these quantities similarly signal the pM-QPT, moving from a minimum to a maximum. These extrema all shift towards $U=0^{+}$(the thermodynamic limit for the metalMott-insulator QPT) as $L$ increases (see the arrows and dotted lines in Fig. 4, left panels). The pM-QPT pulls the final state away from equilibrium as demonstrated by the corresponding increase in $D_{\operatorname{Tr}}\left(\rho_{\tau}, \rho_{\tau}^{\mathrm{eq}}\right)$, which passes from a minimum to a maximum, and dramatically affects the work distribution shape as witnessed by the change in sign of the skewness. After it, as interactions increase further, the final state draws

\footnotetext{
${ }^{5}$ A small trace distance between systems' states guarantees that the other physical properties are also close to the ones of the reference state; the contrary is not always true; however, the behaviors of the trace distance and of a corresponding suitable metric for the local particle density when tracking nonequilibrium dynamics are, in general, similar [56,57].
}

nearer to equilibrium as the system, now almost an insulator, poorly responds to the applied field. Indeed, in this regime, the work distribution comprises very few transitions [Figs. 1(b) and $1(\mathrm{~d})]$.

The value of the trace distance demonstrates that, in the transition region, the final system's state (after the driving) remains always significantly far from equilibrium, ${ }^{6}$ even when the skewness is zero $(U / J \approx 5$ for $L=6$ and 8 ) and the distribution becomes more akin to the linear-response form $\langle W\rangle=\Delta F+\frac{\bar{W}^{2}}{2 k_{B} T}$, which is valid for a close-to-equilibrium dynamics with $\Delta F=\langle W\rangle-\langle\Sigma\rangle / \beta$ the free-energy variation.

While for any $U$, the overall entropy production increases with the system size (see the Supplemental Material [47]), Fig. 4 shows that the entropy production per particle has a complicated dependency on the coupling regime, decreasing for increasing number of particles $L$ in the metal and (quasi) insulating phases, but displaying nonmonotonic behaviorboth with respect to $U$ and to $L-$ in the pM-QPT transition region. The system size affects the work distribution asymmetry in opposite ways before and after the quasi-QPT. Before the pM-QPT, availability of an exponentially increasing number of transitions "regularize" the distribution [compare Figs. 1(a) and 1(c)] contributing to the decrease in its asymmetry, whereas, by de facto restricting the available Hilbert space, the pM-QPT localizes the energy fluctuations in $P(W)$, even for increasing size [compare Figs. 1(b) and 1(d)].

\section{ENTROPY PRODUCTION AND WORK FLUCTUATION-DISSIPATION RELATION}

Close to adiabaticity, classical processes satisfy the work fluctuation-dissipation relation $\langle\Sigma\rangle=\beta^{2} \overline{\mathcal{W}}^{2} / 2[32,33]$; however, recent studies [34] suggest that, for slow quantum processes in open systems, this is governed by the inequality,

$$
\langle\Sigma\rangle \leqslant \beta^{2} \overline{\mathcal{W}}^{2} / 2 \text {. }
$$

We examine the effect of the pM-QPT on the work fluctuationdissipation relation in Fig. 4, right upper panel and show that the transition is marked by a reversing of the inequality (4) with work fluctuations, hence, becoming smaller than dissipation. Most interestingly, after the pM-QPT, whereas increasing $U$ leads the dynamical process back to adiabaticity (Fig. 4, right lower panel, $U>10 J$ ), dissipation remains dominant over work fluctuations, even for very small values of $D_{\operatorname{Tr}}\left(\rho_{\tau}, \rho_{\tau}^{\text {adiab }}\right)$. This reversing of (4) is a many-body effect: The $\mathrm{pM}-\mathrm{QPT}$ dramatically reduces the system response to the

\footnotetext{
${ }^{6}$ The trace distance is bounded $D_{\operatorname{Tr}} \in[0,1]$. Because the trace distance has a maximum, in addition to be sensitive to changes in the system state and continuous, it is possible to establish a quantitative meaning for the words long/short distance: A distance can be defined as short when it is below a certain percentage of the maximum distance [56-58]. For example, a threshold of $10 \%$ corresponds to a short distance being, at least, one order of magnitude shorter than the distance maximum. With this in mind, a distance of $40 \%$ to $60 \%$ of its maximum (as in Fig. 4 of the present paper) shows that the systems are never really close; nevertheless, the variation of the distance between the nonequilibrium and the equilibrium systems with $U$ and $L$ remains substantial.
} 

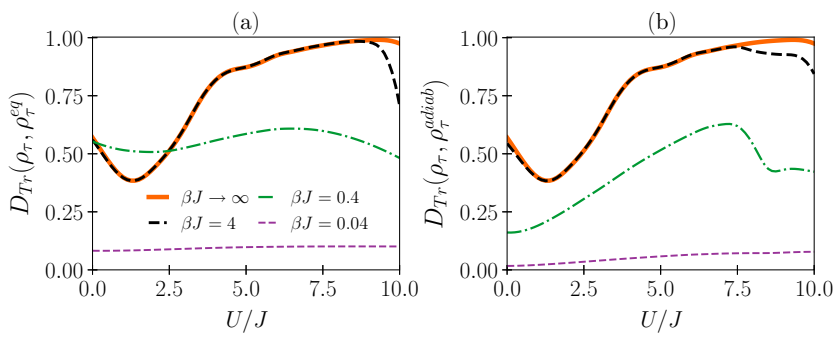

FIG. 5. (a) Trace distance from the equilibrium state and (b) from the adiabatically evolved state as a function of the coupling strength $U / J$ for $L=6$ and at different inverse temperatures $\beta$. The orange line corresponds to the $T=0-\mathrm{K}$ limit for which the initial state is the ground state.

applied field and, hence, the width of the work distribution for all rates of driving, including slow driving (see Fig. 2, middle panels).

In Fig. 5, we analyze the signatures of the quasi-QPT with respect to temperature $(\beta \rightarrow \infty, 4,0.4$, and 0.04$)$ for $D_{\operatorname{Tr}}\left(\rho_{\tau}, \rho_{\tau}^{\text {eq }}\right)$ and $D_{\operatorname{Tr}}\left(\rho_{\tau}, \rho_{\tau}^{\text {adiab }}\right)$. For both distances, these signatures are maximized at the lowest temperatures and washed away by high temperatures. This supports these being signatures of a QPT.

The bottom right panel of Fig. 4 shows that the change in trace distance between the pM-QPT transition region (roughly centered at $U / J=5$ ) and the quasi-insulator phase is of several orders of magnitude: e.g., for $L=8, D_{\mathrm{Tr}} \approx 0.6$ in the transition region and $D_{\mathrm{Tr}}=2 \times 10^{-3}$ for $U / J=20$. Likewise, Fig. 5 shows a variation of $D_{\operatorname{Tr}}$ of two orders of magnitude depending on temperature and coupling strength $U$. We stress that these changes reflect the physics of the system: the formation of an energy gap between states without and with double occupation of sites for increasing $U$ in Fig. 4, and the loss of the pM-QPT transition signature as the temperature increases and the system state has access to the double-occupation high-energy states in Fig. 5.

\section{CONCLUSION}

We discussed the effects of many-body interactions on the statistics of work in inhomogeneous fermionic chains driven for finite times. We considered dynamics from sudden quench to quasi adiabaticity, and observed the signatures of the precursor to the metal-Mott-insulator quantum phase transition. Our results show that, when the system is weakly interacting, the work probability distribution $P(W)$ is highly sensitive to the rate of driving, whereas it remains almost unaffected when many-body interactions are strong.

If the chains' length $L$ is increased and $U / J \lesssim 1, P(W)$ acquires features, such as a well-defined maximum and a bell shape. In contrast, after crossing the precursor to the QPT for $U / J \gtrsim 5$, the work distribution becomes localized at all the explored values of $L$, strongly hindering work extraction with, nonetheless, a price paid in a residual entropy production. The quasi-Mott-insulating phase is associated with a striking reduction of the number of energy transitions arising from the dynamics so that $P(W)$ becomes almost independent of the rate of variation of the external field. This feature leads to entropy production dominating work fluctuations even for slow processes, in contrast to the classical work fluctuationdissipation relation, and at differences with recent predictions for slowly driven open quantum systems.

For dynamics beyond sudden quenches, a change in sign and a remarkable variation in value of the skewness characterize the precursor to the metal-Mott-insulator transition. These features persist even when the number of degrees of freedom is exponentially increased. In the sudden quench regime, the precursor to the QPT affects $P(W)$ only through its effects on the initial and final Hamiltonians' eigenstates; instead, for finite driving times, the precursor to the metal-Mott-insulator transition affects $P(W)$ twice, through its effect on the eigenstates and by modifying the system response to the applied drive. This leads to qualitatively different signatures of the precursor to the QPT on the work distribution, depending on the dynamical regime.

By comparing to the trace distance between the final and the corresponding equilibrium states, we conclude that the third moment of $P(W)$ also retains information about the entropy production and equilibration across the precursor to the QPT.

Experimental realizations of interacting quantum matter could be implemented by means of small molecules and NMR [15,31], coupled quantum dots and ion traps [52,53], or cold atom platforms $[54,55]$. Our findings may help to design timedependent protocols which exploit many-body interactions for, e.g., tailoring work extraction or optimizing efficiency of a refrigeration cycle where the coolant is a strongly interacting many-body system, yielding to novel applications of quantum thermodynamics.

\section{ACKNOWLEDGMENTS}

We thank M. Herrera for very useful discussions during the early stages of the work. We acknowledge financial support from CAPES (Grant No. PDSE-88881.135185/2016-33801); CNPq (Grant: PVE-Processo: 401414/2014-0); FAPESP; UFABC; Instituto Nacional de Ciência Tecnologia em Informação Quâtica (INCT-IQ); Royal Society through the Newton Advanced Fellowship scheme (Grant No. NA140436). K.Z. acknowledges the Schumblenger Foundation for sponsorship through the program Faculty for the Future.
[1] M. Horodecki and J. Oppenheim, Nat. Commun. 4, 2059 (2013).

[2] R. Kosloff, Entropy 15, 2100 (2013).

[3] J. Goold, M. Huber, A. Riera, L. del Rio, and P. Skrzypczyk, J. Phys. A: Math. Theor. 49, 143001 (2016).
[4] S. Vinjanampathy and J. Anders, Contemp. Phys. 57, 545 (2016).

[5] J. Millen and A. Xuereb, New J. Phys. 18, 011002 (2016).

[6] R. Alicki and R. Kosloff, Introduction to Quantum Thermodynamics: History and Prospects (Springer, Cham, Switzerland, 2018), pp. 1-33. 
[7] F. Binder, L. Correa, C. Gogolin, J. Anders, and G. Adesso, Thermodynamics in the Quantum Regime: Fundamental Aspects and New Directions, Fundamental Theories of Physics (Springer, Cham, Switzerland, 2018).

[8] P. Skrzypczyk, A. J. Short, and S. Popescu, Nat. Commun. 5, 4185 (2014).

[9] L. Del Rio, J. Åberg, R. Renner, O. Dahlsten, and V. Vedral, Nature (London) 474, 61 (2011).

[10] M. A. Nielsen and I. L. Chuang, Quantum Computation and Quantum Information (Cambridge University Press, Cambridge, U.K., 2000).

[11] M. Campisi and J. Goold, Phys. Rev. E 95, 062127 (2017).

[12] S. Dorosz, T. Platini, and D. Karevski, Phys. Rev. E 77, 051120 (2008).

[13] T. B. Batalhão, A. M. Souza, L. Mazzola, R. Auccaise, R. S. Sarthour, I. S. Oliveira, J. Goold, G. De Chiara, M. Paternostro, and R. M. Serra, Phys. Rev. Lett. 113, 140601 (2014).

[14] G. T. Landi and D. Karevski, Phys. Rev. E 93, 032122 (2016).

[15] T. B. Batalhão, A. M. Souza, R. S. Sarthour, I. S. Oliveira, M. Paternostro, E. Lutz, and R. M. Serra, Phys. Rev. Lett. 115, 190601 (2015).

[16] D.-T. Hoang, B. P. Venkatesh, S. Han, J. Jo, G. Watanabe, and M.-S. Choi, Sci. Rep. 6, 27603 (2016).

[17] M. Zhong and P. Tong, Phys. Rev. E 91, 032137 (2015).

[18] T. J. G. Apollaro, G. Francica, M. Paternostro, and M. Campisi, Phys. Scr. 2015, 014023 (2015).

[19] C. Jarzynski, H. T. Quan, and S. Rahav, Phys. Rev. X 5, 031038 (2015).

[20] T. Denzler and E. Lutz, Phys. Rev. E 98, 052106 (2018).

[21] A. Sindona, J. Goold, N. L. Gullo, and F. Plastina, New J. Phys. 16, 045013 (2014).

[22] J. Marino and A. Silva, Phys. Rev. B 89, 024303 (2014).

[23] E. Mascarenhas, H. Bragança, R. Dorner, M. França Santos, V. Vedral, K. Modi, and J. Goold, Phys. Rev. E 89, 062103 (2014).

[24] B.-M. Xu, J. Zou, L.-S. Guo, and X.-M. Kong, Phys. Rev. A 97, 052122 (2018).

[25] Q. Wang, D. Cao, and H. T. Quan, Phys. Rev. E 98, 022107 (2018).

[26] J. M. Hickey and S. Genway, Phys. Rev. E 90, 022107 (2014).

[27] D. Schmidtke, L. Knipschild, M. Campisi, R. Steinigeweg, and J. Gemmer, Phys. Rev. E 98, 012123 (2018).

[28] X. Li and Y. Shi, Europhys. Lett. 125, 67003 (2019).

[29] R. Dorner, J. Goold, C. Cormick, M. Paternostro, and V. Vedral, Phys. Rev. Lett. 109, 160601 (2012).

[30] E. G. Arrais, D. A. Wisniacki, A. J. Roncaglia, and F. Toscano, Phys. Rev. E 100, 052136 (2019).

[31] K. Micadei, J. P. Peterson, A. M. Souza, R. S. Sarthour, I. S. Oliveira, G. T. Landi, T. B. Batalhão, R. M. Serra, and E. Lutz, Nat. Commun. 10, 2456 (2019).

[32] J. Hermans, J. Phys. Chem. 95, 9029 (1991).

[33] R. H. Wood, W. C. Muhlbauer, and P. T. Thompson, J. Phys. Chem. 95, 6670 (1991).

[34] H. J. D. Miller, M. Scandi, J. Anders, and M. Perarnau-Llobet, Phys. Rev. Lett. 123, 230603 (2019).

[35] J. H. Drewes, E. Cocchi, L. A. Miller, C. F. Chan, D. Pertot, F. Brennecke, and M. Köhl, Phys. Rev. Lett. 117, 135301 (2016).
[36] J. H. Drewes, L. A. Miller, E. Cocchi, C. F. Chan, N. Wurz, M. Gall, D. Pertot, F. Brennecke, and M. Köhl, Phys. Rev. Lett. 118, 170401 (2017).

[37] M. Boll, T. A. Hilker, G. Salomon, A. Omran, J. Nespolo, L. Pollet, I. Bloch, and C. Gross, Science 353, 1257 (2016).

[38] S. Braun, M. Friesdorf, S. S. Hodgman, M. Schreiber, J. P. Ronzheimer, A. Riera, M. del Rey, I. Bloch, J. Eisert, and U. Schneider, Proc. Natl. Acad. Sci. USA 112, 3641 (2015).

[39] S. Scherg, T. Kohlert, J. Herbrych, J. Stolpp, P. Bordia, U. Schneider, F. Heidrich-Meisner, I. Bloch, and M. Aidelsburger, Phys. Rev. Lett. 121, 130402 (2018).

[40] J. P. Coe, V. V. França, and I. d'Amico, Europhys. Lett. 93, 10001 (2011).

[41] A. D. Greentree, C. Tahan, J. H. Cole, and L. C. Hollenberg, Nat. Phys. 2, 856 (2006).

[42] S. Murmann, A. Bergschneider, V. M. Klinkhamer, G. Zürn, T. Lompe, and S. Jochim, Phys. Rev. Lett. 114, 080402 (2015).

[43] K. Zawadzki, I. D’Amico, and L. N. Oliveira, Brazilian J. Phys. 47, 488 (2017).

[44] D. J. Carrascal, J. Ferrer, J. C. Smith, and K. Burke, J. Phys.: Condens. Matter 27, 393001 (2015).

[45] M. Herrera, R. M. Serra, and I. D'Amico, Sci. Rep. 7, 4655 (2017).

[46] J. R. Johansson, P. D. Nation, and F. Nori, Comput. Phys. Commun. 184, 1234 (2013).

[47] See Supplemental Material at http://link.aps.org/supplemental/ 10.1103/PhysRevResearch.2.033167 for animations of the evolution of $P(W)$ for increasing $\tau$, for fixed $U=0,1 \mathrm{~J}, 2 \mathrm{~J}, \ldots 10 \mathrm{~J}$ and $2 \leqslant L \leqslant 8$; the heatmaps for the average quantum work, and for the second to fourth moments $\langle W-\bar{W}\rangle^{k}, k=2-4$, for all the chains' length considered $(2 \leqslant L \leqslant 8)$; and the heatmaps for the entropy production for $L=4$ and $L=8$.

[48] R. Kawai, J. M. R. Parrondo, and C. Van den Broeck, Phys. Rev. Lett. 98, 080602 (2007).

[49] S. Vaikuntanathan and C. Jarzynski, Europhys. Lett. 87, 60005 (2009).

[50] J. M. R. Parrondo, C. V. den Broeck, and R. Kawai, New J. Phys. 11, 073008 (2009).

[51] M. Wilde, Quantum Information Theory, Quantum Information Theory (Cambridge University Press, Cambridge, New York, 2013).

[52] J. Roßnagel, S. T. Dawkins, K. N. Tolazzi, O. Abah, E. Lutz, F. Schmidt-Kaler, and K. Singer, Science 352, 325 (2016).

[53] S. An, J.-N. Zhang, M. Um, D. Lv, Y. Lu, J. Zhang, Z.-Q. Yin, H. Quan, and K. Kim, Nat. Phys. 11, 193 (2015).

[54] G. D. Chiara, A. J. Roncaglia, and J. P. Paz, New J. Phys. 17, 035004 (2015).

[55] F. Cerisola, Y. Margalit, S. Machluf, A. J. Roncaglia, J. P. Paz, and R. Folman, Nat. Commun. 8, 1241 (2017).

[56] A. H. Skelt, R. W. Godby, and I. D’Amico, Phys. Rev. A 98, 012104 (2018).

[57] A. H. Skelt and I. D'Amico, Adv. Quantum Technol. 3, 1900139 (2020).

[58] S. Marocchi, S. Pittalis, and I. D’Amico, Phys. Rev. Mater. 1, 043801 (2017). 